\title{
A METHOD FOR THE DETECTION OF EPISTASIS IN CHROMOSOME SUBSTITUTION LINES OF HEXAPLOID WHEAT
}

\author{
J. W. SNAPE, C. N. LAW and A. J. WORLAND \\ Plant Breeding Institute, Maris Lane, Trumpington, Cambridge CB2 2LQ
}

Received 16.ix.74

\section{Summary}

\begin{abstract}
A crossing procedure, analogous to the triple test cross of Kearsey and Jinks (1968), is described for detecting epistasis in a series of single chromosome substitution lines of hexaploid wheat. By applying scaling tests to the generation means produced, it is possible to identify the interacting chromosomes and also residual " background " interactions. The analysis of second degree statistics can, under certain circumstances, allow the types of interaction present to be identified.

The method is illustrated using the complete set of 21 single chromosome substitution lines of the wheat variety Cappelle-Desprez into the variety Chinese Spring.
\end{abstract}

\section{INTRODUGTION}

Bread wheat, Triticum aestivum $(2 n=6 x=42)$, is able to tolerate chromosome loss because of its hexaploid nature. This has enabled the development of aneuploid stocks and cytological techniques which enable single pairs of chromosomes to be transferred from one variety to another.

Law (1966, 1968, 1972a) and Aksell (1967) have described methods using such chromosome substitution lines of hexaploid wheat whereby genetical differences for quantitative characters can be analysed in detail. In particular, they have described two types of diallel cross, named the Triparental crosses 1 and 2 which enable the additive, non-additive and interaction effects of whole chromosomes to be detected. However, only in the absence of epistasis (or more correctly non-homologous chromosome interactions) can the magnitude of the additive and non-additive (dominance) effects be unambiguously estimated, and this, in turn, restricts the predictive value of the agronomic benefit of known chromosome transfers, without more detailed genetic analysis.

The present paper describes a test for epistasis which is an extension of the Triparental cross 2 by the inclusion of the cross between the substitution line and the $F_{1}$ between the donor and recipient lines. The addition of this cross is equivalent to the triple-test cross of Kearsey and Jinks (1968) and allows an independent test of epistasis for each line and an indication of the types of interaction, if any, present in the whole set of substitution lines.

\section{THEORY}

(i) Scaling tests

A set of $n$ single chromosome substitution lines of one variety, termed the Donor $(D)$, into another variety, termed the Recipient $(R)$ are crossed 
to the Donor, Recipient and the $\mathrm{F}_{1}(R D)$ between them. If $n$ is equal to the 21 homologous chromosome pairs of hexaploid wheat then a complete set of single chromosome substitution lines is, of course, being considered.

The mean genetical expression of these families can be described using the notation of Law (1972a) after Hayman and Mather (1955), and it is easily shown, in the absence of epistasis, that for each substitution line:

$$
\left(\overline{S_{i} \times D}\right)+\left(\overline{S_{i} \times R}\right)-2\left(\overline{S_{i} \times R D}\right)=0 \text { where } i=1 \text { to } n \text {. }
$$

This is, of course, a scaling test and can be tested for significance against its standard error:

$$
\sqrt{V_{\left(S_{i} \overline{\times} D\right)}+V_{\left(S_{i} \times R\right)}+4 V_{\left(S_{i} \times R D\right)}}
$$

This allows independent tests of epistasis in the crosses involving each of the substitution lines.

If, for example, there are only three chromosomes A, B and C with theireffects in association, then, if epistasis is present, and assuming only dichromosomal interactions the above is an estimate of:

$\frac{1}{2} i_{a b}+\frac{1}{2} i_{a c}+\frac{1}{2} i_{b c}+\frac{1}{2} j_{a b}-\frac{1}{2} j_{b a}+\frac{1}{2} j_{a c}-\frac{1}{2} j_{c a}+\frac{1}{2} j_{b c}+\frac{1}{2} j_{c b}-\frac{1}{2} l_{a b}-\frac{1}{2} l_{a c}+\frac{1}{2} l_{b c}$ for the crosses involving substitution line $A A b b c c$.

If chromosome $\mathrm{A}$ was in the dispersed condition, the Recipient being $A A b b c c$ and the substitution line $a a b b c c$, then the estimate becomes:

$$
-\frac{1}{2} i_{a b}-\frac{1}{2} i_{a c}+\frac{1}{2} i_{b c}-\frac{1}{2} j_{a b}-\frac{1}{2} j_{b a}-\frac{1}{2} j_{a c}-\frac{1}{2} j_{c a}+\frac{1}{2} j_{b c}-\frac{1}{2} j_{c b}-\frac{1}{2} l_{a b}-\frac{1}{2} l_{a c}+\frac{1}{2} l_{b c} .
$$

This test provides an estimate, therefore, of the sum of the interactions of the substituted chromosome with other chromosomes of the complement plus the sum of the interactions between other chromosomes of the complement.

In common with the Triparental crosses 1 and 2 tests for epistasis, the efficiency of the present test in detecting interaction, when present, will depend on the degree of association and direction of the effects of the interacting chromosomes and on the type of interaction, whether predominantly of a complementary or duplicate nature (Law, 1972a).

The Triparental cross 2 described by Law (loc. cit.) and Aksell (loc. cit.) detects only the interactions of the substituted chromosome with the other chromosomes in the background. This test may be combined with the present test to detect " background" interactions.

In the absence of such interactions

$$
\frac{1}{2}\left(\overline{S_{i} \times D}\right)+\frac{1}{2}\left(\overline{S_{i} \times R}\right)+\frac{1}{2} \bar{S}_{i}+\frac{1}{2} \overline{R D}-2\left(S_{i} \overline{\times} R D\right)=0
$$

with a variance of

$$
\frac{1}{4} V_{\left(S_{i} \times D\right)}+\frac{1}{4} V_{\left(S_{i} \times R\right)}+\frac{1}{4} V_{\bar{S}_{i}}+\frac{1}{4} V_{(\overline{R D})}+4 V_{\left(S_{i} \times\right.}{ }_{R D)} .
$$

If interaction is present, the above is an estimate, irrespective of whether the substituted chromosome is dispersed or associated, of:

$$
\frac{1}{2}\left(i_{b c}+j_{b c}+j_{c b}+l_{b c}\right)
$$


Thus with the set of crosses described here, three tests of epistasis are possible. These are:

(i) Triple Test Cross: $\overrightarrow{S_{i} R}+\overrightarrow{S_{i} D}-2\left(\overrightarrow{S_{i} \times R D}\right)$

(ii) Triparental Cross 2: $\frac{1}{2}\left(\overline{S_{i} R}+\overline{S_{i} D}-\bar{S}_{i}-\overline{R D}\right)$

(iii) "Background" effects: $\frac{1}{2}\left(\overline{S_{i} R}+\overline{S_{i} D}+\bar{S}_{i}+\overline{R D}-4\left(\overline{S_{i} \times R D}\right)\right)$.

It is easily shown that tests (ii) and (iii) are orthogonal but not tests (i) and (ii) or (i) and (iii).

If epistasis is present the Triparental cross 2 values will indicate which chromosomes are the most interactive. In the absence of epistasis the additive and non-additive effects of the chromosomes can be estimated as shown by Law $(1972 a)$.

(ii) Second degree statistics

Since each value of $\overline{S_{i} R}+\overline{S_{i} D}-2\left(\overline{S_{i} \times R D}\right)$ is independent, the variance of these values can, under certain conditions, indicate the type of interactions present in the substitution series.

Assuming, only three interacting chromosomes in the set, dichromosomal interactions only, and associated chromosomes, then the expectations for $\overline{S_{i} R}+\overline{S_{i} D}-2\left(\overline{S_{i} \times R D}\right)$ are as shown in table 1 .

Following Jinks and Perkins (1970), it can be shown that the overall sum of squares: $\left.\sum_{i=1}^{n} \overline{\left(S_{i} R\right.}+\overline{S_{i} D}-2\left(S_{i} \times R D\right)\right)^{2} / 6$ for $n$ d.f. detects variation due to $i, j$ and $l$ type interactions. However, the variation of the individual values around the overall mean allows a test of $j+l$ type interactions only and gives a sum of squares for $(n-1)$ d.f. of

$$
\sum_{i=1}^{n}\left\{\left(\overline{S_{i} R}+\overline{S_{i} D}-2 \overline{\left(S_{i} \times R D\right.}\right)\right\}^{2} / 6-\left\{\sum_{i=1}^{n}\left(\overline{S_{i} R}+\overline{S_{i} D}-2\left(\overline{S_{i} \times R D}\right)\right)\right\}^{2} / 6 n .
$$

In the absence of these interactions the overall mean contains terms only in $\frac{1}{2} i$ and thus gives a sum of squares for 1 d.f. which detects this type of interaction. If $l$ and $j$ type interactions are present then this term is, of course, a function of all three types of interaction and only $j+l$ type interactions can be unambiguously detected, although not separated from one another, unlike the test of Jinks and Perkins (loc. cit.) where $i$ interactions can be unambiguously detected in the presence of $j$ and $l$ type interactions.

\section{EXAmple}

This concerns an experiment carried out at the Plant Breeding Institute, Cambridge, involving the complete set of 21 single chromosome substitution lines of the winter wheat variety Cappelle-Desprez (Donor) into the variety Chinese Spring (Recipient).

The experiment was sown as a randomised plot experiment in 1972, and the character under consideration here is final plant height.

The results of the three scaling tests for epistasis are shown in table 2 and indicate that epistasis is present in the genetical control of this character. 


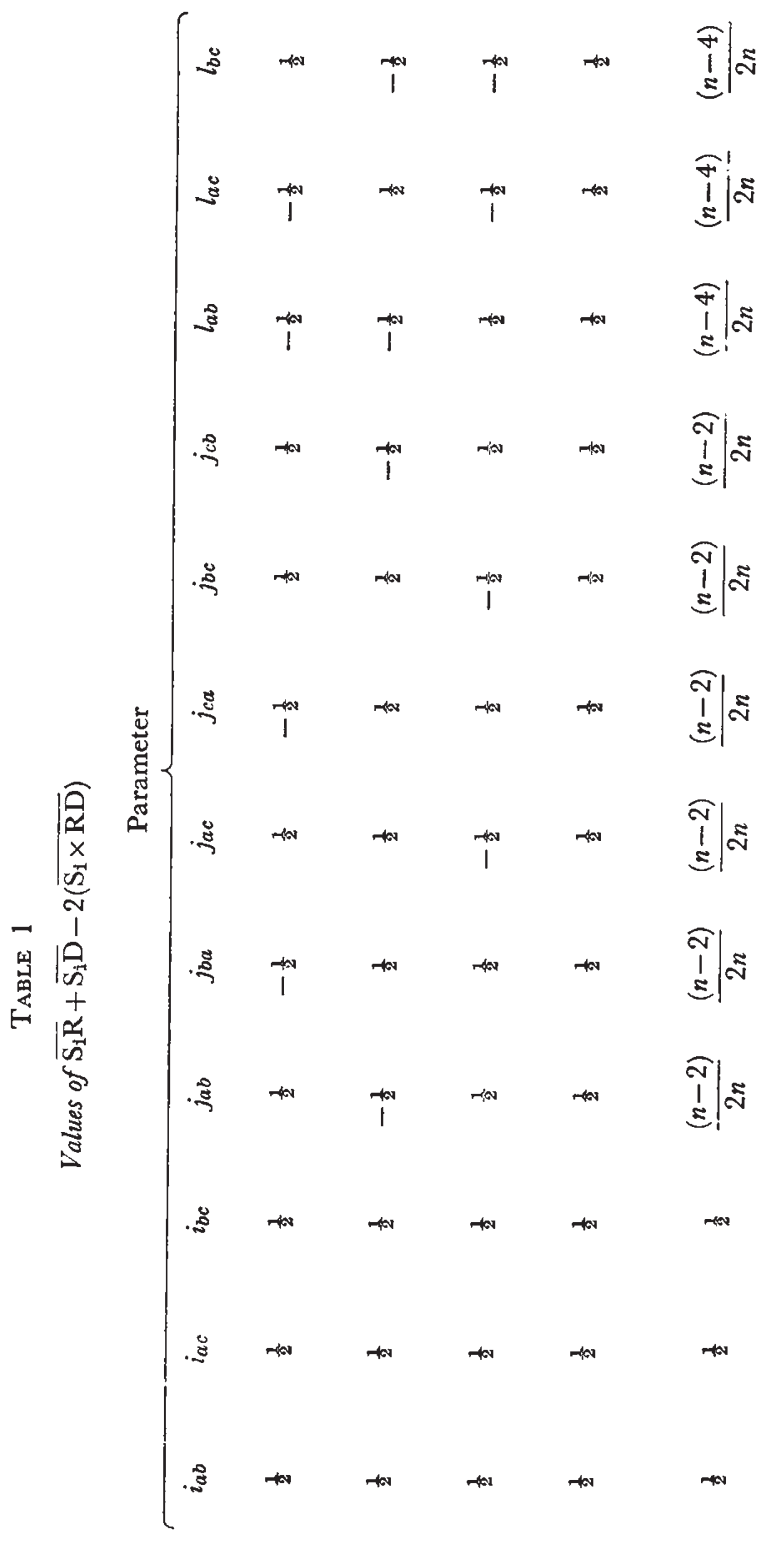

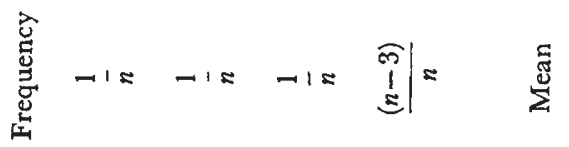

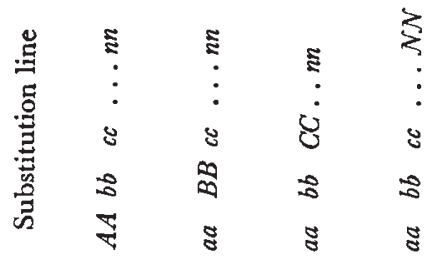


All significant estimates of tests (i) and (iii) are negative, and indeed, most of the non-significant estimates are also negative. Thus the interactions present are, in general, acting to reduce plant height. Since, on evidence to be presented later, the dominance $(h)$ effects are predominantly positive, then a predominantly duplicate type of gene interaction is involved in the genetical control of this character (Mather, 1967). Interestingly, the substitution lines of the variety Hope into Chinese Spring also showed this type of genetical architecture (Law and Worland, 1972b) which was related to the hexaploid nature of the wheat genotype and the functional identity of chromosomes within homoeologous groups. However, such a relationship cannot be established with certainty here. Indeed, the net

TABle 2

Results of scaling tests

\begin{tabular}{cccl} 
Chromosome & Test (i) & Test (ii) & Test (iii) \\
1A & -4.04 & 0.91 & -4.95 \\
1B & -3.91 & $-2.23 *$ & -1.68 \\
1D & $-10.66^{*}$ & -2.66 & $-8.00^{*}$ \\
2A & -3.72 & 0.15 & -3.87 \\
2B & -1.56 & 0.42 & -1.98 \\
2D & -1.91 & 1.95 & -3.86 \\
3A & 3.16 & 1.22 & 1.94 \\
3B & -6.99 & -1.38 & -5.61 \\
3D & -3.63 & 1.94 & -5.57 \\
4A & -5.11 & -1.56 & -3.55 \\
4B & 1.01 & -0.31 & 1.32 \\
4D & -1.42 & 2.65 & -4.07 \\
5A & -1.03 & 2.40 & -3.43 \\
5B & -4.41 & -1.63 & -2.78 \\
5D & $-9.14 *$ & -0.63 & $-8.51 *$ \\
6A & 0.35 & 0.60 & -0.25 \\
6B & -3.09 & $7.28 * *$ & $-10.37 * *$ \\
6D & -4.75 & -2.00 & $-6.75 *$ \\
7A & -4.54 & 1.36 & $-5.90 *$ \\
7B & -4.21 & 2.08 & $-6.29 *$ \\
7D & -1.42 & 1.32 & -2.74 \\
\multicolumn{4}{c}{} \\
Probability $*=0.05-0.01 ; * *=0.01-0.001$.
\end{tabular}

interactions of individual chromosomes (test (ii)) are ambidirectional in nature in the Cappelle-Desprez substitutions and of the two significant effects, those of chromosomes $1 \mathrm{~B}$ and $6 \mathrm{~B}$, the former is negative and the latter, positive. It would appear, therefore, that the net estimates of interaction in these crosses are made up of both positive and negative effects but with a preponderance of the latter.

Turning to the results of the triple test cross for epistasis, only two estimates are significant, those involving crosses of the substitution lines of chromosomes 1D and 5D. Neither of these chromosomes is detected as being individually interactive. In fact, these two effects are mainly the result of significant background interactions which are reinforced by the effects of the interactions of chromosomes $1 \mathrm{D}$ and $5 \mathrm{D}$. In contrast, the large positive effect of chromosome $6 \mathrm{~B}$ is opposed by the negative interactions in the background and a non-significant estimate is obtained. 
The analysis of variance with respect to the variation of the values of $\overline{S_{i} R}+\overline{S_{i} D}-2\left(\overline{S_{i} \times R D}\right)$ is shown in table 3 .

In this analysis, the "total epistasis" item is not significant. However, the important comparisons are obtained on partitioning this item. Thus although the "residual epistasis" item is not significant, the "overall epistasis" item is highly significant. Since the former detects $j+l$ type interactions and the latter $i+j+l$ type interactions, then the interactions present must be predominantly of the $i$ type in this series of crosses.

The above conclusions are reinforced by the analysis of the generation means of progeny produced by crossing the Donor and Recipient varieties. Six generations are available, the two parents, their $F_{1}, F_{2}$ and backcrosses.

The result of the $C$ scaling test (Mather and Jinks, 1971) is $4 \cdot 65 \pm 0.92$. This is highly significant indicating that epistasis is present. This is further

TABLE 3

\begin{tabular}{|c|c|c|c|c|}
\hline \multicolumn{5}{|c|}{ Analysis of variance of $\overline{\mathrm{S}_{1} \mathrm{R}}+\overline{\mathrm{S}_{1} \mathrm{D}}-2\left(\overline{\mathrm{S}_{1} \times \mathrm{RD}}\right)$ values } \\
\hline Item & d.f. & M.S. & V.R. & $\mathbf{P}$ \\
\hline $\begin{array}{l}\text { "Total Epistasis" } \\
\text { " Overall epistasis" }(i+j+l) \\
\text { " Residual epistasis" }(j+l)\end{array}$ & $\begin{array}{r}21 \\
1 \\
20\end{array}$ & $\begin{array}{r}3 \cdot 516 \\
40 \cdot 030 \\
1 \cdot 690\end{array}$ & $\begin{array}{r}1 \cdot 30 \\
14 \cdot 83 \\
0 \cdot 63\end{array}$ & $\begin{array}{l}17 \% \\
<0 \cdot 1 \% \\
90 \%\end{array}$ \\
\hline Replicates & 539 & $2 \cdot 699$ & & \\
\hline
\end{tabular}

TABLE 4

Analysis of generation means

\begin{tabular}{|c|c|}
\hline Model 1 & Model 2 \\
\hline$\hat{m}=107 \cdot 38 * * *$ & $\hat{m}=114 \cdot 72 * * *$ \\
\hline$\{\hat{d}\}=2.58$ & $\{\hat{d}\}=2 \cdot 20$ \\
\hline$\{\hat{h}\}=13.54 * * *$ & $\begin{array}{l}\{\hat{h}\}=4 \cdot 62 * \\
\{\hat{i}\}=-9 \cdot 30 * * *\end{array}$ \\
\hline$\chi_{\left[{ }^{3}\right]}^{2}=26 \cdot 6 * * *$ & $\chi_{[2]}^{2}=0.85$ \\
\hline
\end{tabular}

illustrated by the failure of an $m,\{d\}$ and $\{h\}$ model to account for the observed statistics-Model 1, table 4 . However by allowing for additive $\times$ additive (or $i$ ) interactions-Model 2, table 4, a satisfactory fit is obtained. Furthermore, the value of $\{\hat{i}\}$ is negative, as would be expected from the above, and $\{\hat{h}\}$ positive, suggesting that a duplicate type of gene interaction is present.

In conclusion, the analysis of first- and second-degree statistics of the present experiment demonstrate that epistasis is involved in the genetical control of final height in this set of substitution lines. Thus a more detailed genetic analysis, to estimate the individual additive, dominance and interaction parameters of each chromosome, is needed before prediction of the effects of new chromosomal combinations can be attempted. Many chromosomes contribute to the "net" interaction in these crosses particularly chromosomes $\mathrm{IB}$ and $6 \mathrm{~B}$, and these interactions are mainly of an additive $\times$ additive type. Because the interactions are of this type, they are, of course, fixable in inbred lines which can be derived from a cross 
between the donor and recipient varieties. However, although the interactions of different chromosomes are ambidirectional in nature there is a preponderance of effects opposing the additive and dominance effects of the chromosomes, a genetical situation corresponding to the duplicate gene interaction situation of classical Mendelian genetics. This type of gene action will restrict the expression of transgressive segregation for increased height in the $F_{2}$ and $F_{3}$ generations derived from the cross between these two varieties.

\section{REFERENGES}

AKSELL, R. 1967. Quantitative genetic analysis of characters in wheat using chromosome substitution lines (Theoretical considerations). Genetics, 57, 195-211.

HAYMAN, B. I., AND MATHER, K. 1955. The description of genic interactions in continuous variation. Biometrics, 2, 69-82.

JINKS, J. L., AND PERKINS, J. M. 1970. A general method for the detection of additive dominance and epistatic components of variation. III. $\mathrm{F}_{2}$ and backcross populations. Heredity, 25, 419-429.

KEARSEY, M. J., AND JINKS, J. L. 1968. A general method of detecting additive, dominance and epistatic variation for metrical traits. I. Theory. Heredity, 23, 403-409.

LAw, c. N. 1966. Biometrical analysis using chromosome substitutions within a species. In Chromosome Manipulation in Plant Genetics (Eds. R. Riley and K. R. Lewis). Suppt. Heredity, 20, 59-85.

LAW, c. N. 1968. Genetic analysis using inter-varietal chromosome substitutions. Proc. 3rd Int. Wheat. Genet. Symp., Canberra, 331-342.

LAW, C. N. 1972a. The analysis of inter-varietal chromosome substitutions in wheat and their first generation hybrids. Heredity, 28, 169-179.

LAW, C. N. AND WORLAND, A. J. 1972b. Aneuploidy in wheat and its uses in genetic analysis. Rep. Plant Breed. Inst., Cambridge, 1972, 25-65.

MATHER, к. 1967. Complementary and duplicate gene interactions in biometrical genetics. Heredity, 22, 97-102.

MATHER, K., AND JiNKS, J. L. 1971. Biometrical Genetics. Chapman and Hall Ltd., London. 